\title{
Modelling heat transfer in steel coils
}

\author{
Steven I. Barry ${ }^{1} \quad$ Winston L. Sweatman ${ }^{2}$
}

(Received 13 August 2008; revised 12 November 2008)

\begin{abstract}
During annealing, heat transfer within steel coils is complicated by the different conductivity in radial and axial directions due to small air gaps between the steel layers in the radial direction. Here an analytic solution augmented with numerical calculations illustrates some of the fundamental behaviour of the system, including calculations of the time lag. The beneficial effects of additional heating on the curved surfaces are shown.
\end{abstract}

\section{Contents}

\section{Introduction} 1446-8735. (Print two pages per sheet of paper.) 
4 Results

C675

5 Conclusion

C678

References

C680

\section{Introduction}

Annealing of steel coils is done to alter the crystal structure of the steel which can become brittle during cold rolling. It requires heating the coil to a high temperature $\left(\approx 700^{\circ} \mathrm{C}\right)$ and maintaining this temperature for a significant period of time. Initially, the entire coil must be raised to the prescribed temperature and so the duration of the initial heating is determined by the part of the coil which is slowest to heat: the cold point. We call the time required to heat the cold point to the annealing temperature the time lag. Overall the aim is to heat the coil for the minimum possible time to save energy costs. However, if the cold point is insufficiently annealed then it is expensive to reprocess the steel coil. Hence accurate prediction of the correct annealing time is desirable.

This research arose from a recent Mathematics in Industry Study Group problem, held at University of Wollongong in February 2008. This project was sponsored by New Zealand Steel and concerned the specific characteristics of a Uniflow Annealing System (UAS) furnace as used in Auckland. The proceedings of that meeting [7] provide more details of the modelling of the process and an introduction to our analytic approach. Here we further explore the properties and behaviour of the system as predicted by the model and compare the existing heating process to one with additional heating on the curved surfaces. Calculations of the time lag-the time to reach a required temperature - are also given. 


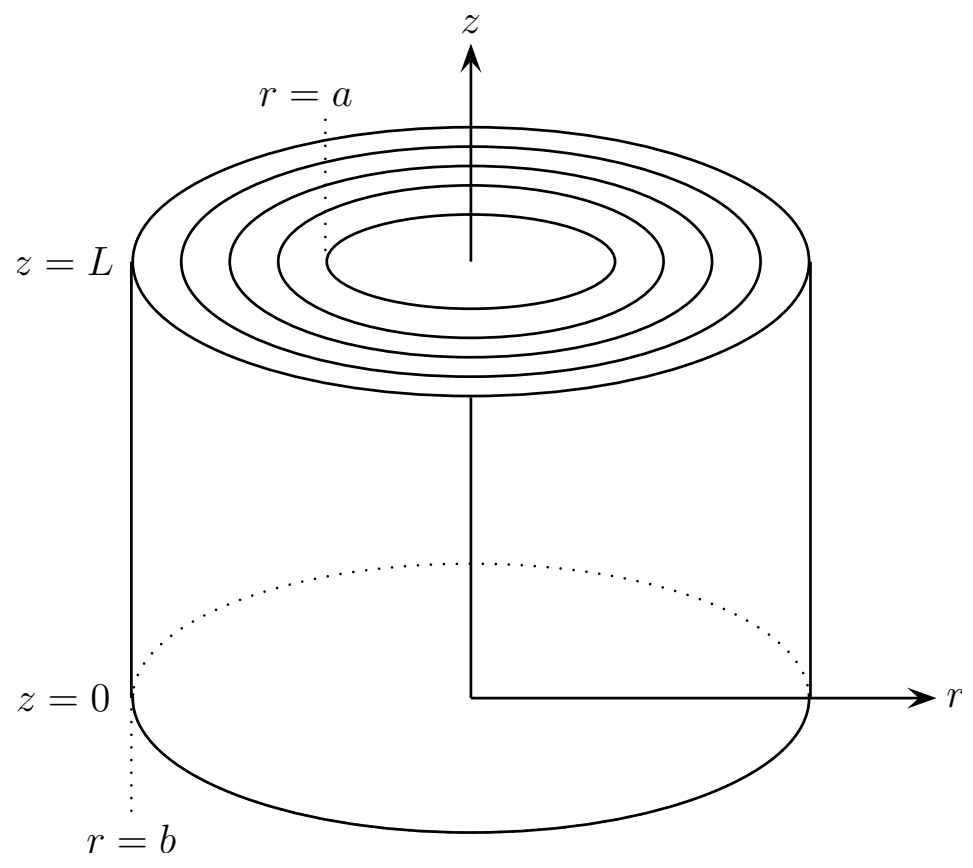

Figure 1: Schematic of the coil as nested cylinders.

\section{$2 \quad$ Model of the steel coil}

The coils are formed by cylindrically wrapping a sheet of steel about an armature which is afterwards removed. They are hollow cylinders of approximately 10-20 tonnes in weight, a metre in height and diameter, and with a steel thickness of $0.4-3 \mathrm{~mm}$. A schematic of the geometry is given in Figure 1. Calculating the heat transfer within the coil is complicated by air gaps between the layers of steel; there is reduced conductance due to contact surface roughness even where the layers are in direct contact [9]. There is a dependence on the winding coil tension, which varies with curved position, and the heat expansion of the coils [10]. The air gap is also larger at the 
vertical ends of the coil due to nonuniformity of the rolling process.

A common approach $[2,4,6,10,11,12]$ assumes that, since the number of layers is large, the interior of the coil can be treated as a uniform material with anisotropic conductivity: the radial conductivity being dependent on position. Taking $r$ and $z$ as radial and vertical spatial coordinates, $t$ as time, $T(r, z, t)$ as temperature, $c_{p}(T)$ as heat capacity, $\rho(T)$ as steel density, and $k_{r}(r, z, t)$ and $k_{z}$ radial and vertical conductivities, we have the anisotropic diffusion equation

$$
\frac{\partial\left(c_{p} \rho T\right)}{\partial t}=\frac{1}{r} \frac{\partial}{\partial r}\left(k_{r} r \frac{\partial T}{\partial r}\right)+\frac{\partial}{\partial z}\left(k_{z} \frac{\partial T}{\partial z}\right) .
$$

The vertical conductivity $k_{z}$ is usually assumed to be the conductivity of steel $k_{\mathrm{s}}$ but finding an appropriate relationship for $k_{r}(r, z, t)$ is more challenging [10]. For the present model we do not analyse all the possible influences upon $k_{r}$ which include winding tension [3], contact resistances [9] and thermal expansion [10]. Rather, we present solutions for constant conductivities which gives the dominant behaviour and estimates of heating times. Additionally these solutions provide checks of more complicated numerical schemes.

We consider three sets of boundary conditions. In the UAS furnace each coil is placed upon its flat end on a perforated metal sheet and heated by a combination of direct radiation from above and conduction from hot gas moving around the coil. As argued by McGuinness et al. [7], a simple model for this system imposes the ambient gas temperature on the flat top and base of the coil to allow for rapid effective heating by radiation and conduction. In contrast, the curved cylindrical surfaces are believed to be subject to Newton heating from the hot gas. We also consider alternative scenarios where the curved surfaces are more effectively heated allowing them also to be modelled as being at the imposed ambient temperature. 


\section{$3 \quad$ Linear solution}

Here we investigate analytic solutions for linear heat transport within a coil modelled as a homogeneous hollow cylinder with anisotropic heat conductance. The cylinder is defined radially for $r \in[a, b]$ and vertically for $z \in$ $[0,1]$ (compare with Figure 1). Assuming constant values of $D_{r}=k_{r} /\left(\rho c_{p}\right)$ and $D_{z}=k_{z} /\left(\rho c_{p}\right)$, and denoting the temperature of the external gas $T_{g}$ and the initial temperature of the coil $\mathrm{T}_{0}$, Equation (1) simplifies to

$$
\frac{\partial T}{\partial t}=D_{r} \frac{1}{r} \frac{\partial}{\partial r}\left(r \frac{\partial T}{\partial r}\right)+D_{z} \frac{\partial}{\partial z}\left(\frac{\partial T}{\partial z}\right)
$$

with

$$
\mathrm{T}(\mathrm{r}, z=0, \mathrm{t})=\mathrm{T}(\mathrm{r}, z=\mathrm{L}, \mathrm{t})=\mathrm{T}_{\mathrm{g}}, \quad \mathrm{T}(\mathrm{r}, z, \mathrm{t}=0)=\mathrm{T}_{0} .
$$

We consider three possible boundary conditions for the curved surfaces, referred to as BC1-BC3:

$$
\begin{array}{ll}
\text { BC1: } & {\left[k_{r} \frac{\partial T}{\partial r}-H\left(T-T_{g}\right)\right]_{r=a}=\left[k_{r} \frac{\partial T}{\partial r}+H\left(T-T_{g}\right)\right]_{r=b}=0,} \\
\text { BC2: } & {\left[k_{r} \frac{\partial T}{\partial r}-H\left(T-T_{g}\right)\right]_{r=a}=\left[T-T_{g}\right]_{r=b}=0,} \\
\text { BC3: } & {\left[T-T_{g}\right]_{r=a}=\left[T-T_{g}\right]_{r=b}=0,}
\end{array}
$$

where $\mathrm{H}\left[\mathrm{J} / \mathrm{m}^{2} / \mathrm{s} / \mathrm{K}\right]$ is the heat transfer coefficient for Newton heating. These boundary conditions correspond to different combinations of Newton heating and imposed temperatures on the inner and outer curved surfaces. Thus BC1 corresponds to the situation in the UAS furnace studied by McGuinness et al. [7], with Newton heating on both curved surfaces. For BC2 the outer curved surface is maintained at ambient gas temperature as might be achieved with additional direct radiant heating. The condition BC3 arises if the entire external surface of the coil is subject to an imposed temperature.

Equations (2-6) are re-scaled

$$
\mathrm{t}=\mathrm{t}_{\mathrm{o}} \tau, \quad \mathrm{r}=\mathrm{br}^{*}, \quad z=\mathrm{L} z^{*}, \quad \mathrm{u}=\frac{\mathrm{T}-\mathrm{T}_{\mathrm{g}}}{\mathrm{T}_{\mathrm{o}}-\mathrm{T}_{\mathrm{g}}},
$$


where $r^{*}, z^{*}, \tau$ and $u$ are the nondimensional variables. The time scale is chosen as $t_{0}=\mathrm{L}^{2} / \mathrm{D}_{z}$. For convenience, we drop the ${ }^{*}$ notation, assign relative diffusivity $D=D_{r} L^{2} / D_{z} b^{2}$, set $h=H b / k_{r}$ and now use $\alpha=a / b \in$ $[0,1]$ to represent the ratio of original lengths. The new nondimensional system is

$$
\begin{aligned}
& \frac{\partial u}{\partial \tau}=D \frac{1}{r} \frac{\partial}{\partial r}\left(r \frac{\partial u}{\partial r}\right)+\frac{\partial^{2} u}{\partial z^{2}}, \\
& u(r, z=0, \tau)=u(r, z=1, \tau)=0, \quad u(r, z, \tau=0)=1 .
\end{aligned}
$$

The boundary conditions, Equations (4)-(6) are replaced by the appropriate conditions $\frac{\partial u}{\partial r}= \pm$ hu or $u=0$.

The solutions are found using separation and Sturm-Liouville theory. Separating $u$ as $u(r, z, \tau)=R(r) Z(z) T(\tau)$ gives

$$
\begin{aligned}
\mathrm{Z}^{\prime \prime} & =\mu \mathrm{Z}, \quad \mathrm{Z}(0)=\mathrm{Z}(1)=0, \\
\mathrm{~T}^{\prime} & =(\mathrm{D} \omega+\mu) \mathrm{T}, \\
\mathrm{R}^{\prime \prime}+\frac{1}{r} \mathrm{R}^{\prime} & =\omega \mathrm{R} .
\end{aligned}
$$

with $\omega$ and $\mu$ the eigenvalues. The eigenfunctions are

$$
\begin{aligned}
& Z=\sin n \pi z, \quad \mu=-(n \pi)^{2}, \quad n=1,2, \ldots, \\
& R=C_{0}(\lambda r) \equiv J_{0}(\lambda r)+B Y_{0}(\lambda r), \quad \omega=-\lambda^{2}, \\
& T=\exp \left\{-\left[D \lambda^{2}+(n \pi)^{2}\right] \tau\right\},
\end{aligned}
$$

where $\mathrm{B}$ is a constant, and $\mathrm{J}_{0}$ and $\mathrm{Y}_{0}$ are Bessel functions. The full solution is

$$
u(r, z, \tau)=\sum_{n=1}^{\infty} \sum_{m=1}^{\infty} A_{m n} e^{-\left[D \lambda_{m}^{2}+(n \pi)^{2}\right] \tau} \sin n \pi z C_{0}\left(\lambda_{m} r\right)
$$

The constants $A_{m n}$ are found using Sturm-Liouville orthogonality to be $[1$, ch 9,11$]$

$$
A_{m n}=\frac{\int_{0}^{1} \sin (n \pi z) d z}{\int_{0}^{1} \sin ^{2}(n \pi z) d z} \frac{\int_{\alpha}^{1} r C_{0}\left(\lambda_{m} r\right) d r}{\int_{\alpha}^{1} r C_{0}^{2}\left(\lambda_{m} r\right) d r}
$$




$$
=2 \frac{1-(-1)^{n}}{n \pi} \frac{\left[\frac{r}{\lambda_{m}} C_{1}\left(\lambda_{m} r\right)\right]_{\alpha}^{1}}{\left[\frac{r^{2}}{2}\left(C_{0}^{2}\left(\lambda_{m} r\right)+C_{1}^{2}\left(\lambda_{m} r\right)\right)\right]_{\alpha}^{1}},
$$

where $\mathrm{C}_{0}$ and $\mathrm{C}_{1}$ are defined, as in Equation (14), by

$$
\begin{aligned}
& \mathrm{C}_{0}(\mathrm{x})=\mathrm{J}_{0}(\mathrm{x})+\mathrm{BY}_{0}(\mathrm{x}), \\
& \mathrm{C}_{1}(\mathrm{x})=\mathrm{J}_{1}(\mathrm{x})+\mathrm{BY}_{1}(\mathrm{x}) .
\end{aligned}
$$

Note that $C_{1}^{2}(x) \equiv\left[C_{1}(x)\right]^{2}$.

The dominant behaviour of Equation (16) is given by the leading eigenvalues $\lambda_{1}$ and $\pi$ and their corresponding eigenfunctions. The eigenvalues $\lambda_{m}$ and the constant B in Equation (14) are still to be determined.

For $B C 1, R^{\prime}(\alpha)=h R(\alpha)$ and $R^{\prime}(1)=-h R(1)$, giving

$$
\begin{aligned}
-\lambda J_{1}(\lambda \alpha)-B \lambda Y_{1}(\lambda \alpha)-h J_{0}(\lambda \alpha)-h B Y_{0}(\lambda \alpha) & =0 \\
-\lambda J_{1}(\lambda)-B \lambda Y_{1}(\lambda)+h J_{0}(\lambda)+h B Y_{0}(\lambda) & =0 .
\end{aligned}
$$

The infinite number of values for $\lambda=\lambda_{m}, m=1,2, \ldots$, occur when Equations (21) and (22) have a consistent solution for B; that is, when

$$
\left|\begin{array}{cc}
\lambda J_{1}(\lambda \alpha)+h J_{0}(\lambda \alpha) & \lambda Y_{1}(\lambda \alpha)+h Y_{0}(\lambda \alpha) \\
-\lambda J_{1}(\lambda)+h J_{0}(\lambda) & -\lambda Y_{1}(\lambda)+h Y_{0}(\lambda)
\end{array}\right|=0,
$$

which is solved numerically for $\lambda_{m}$. Equation (21) gives

$$
B=-\frac{h J_{0}(\lambda)-\lambda J_{1}(\lambda)}{h Y_{0}(\lambda)-\lambda Y_{1}(\lambda)}
$$

Similarly for $\mathrm{BC} 2, \mathrm{R}^{\prime}(\alpha)=h \mathrm{R}(\alpha)$ and $\mathrm{R}(1)=0$ giving

$$
\left|\begin{array}{cc}
\lambda \mathrm{J}_{1}(\lambda \alpha)+h \mathrm{~J}_{0}(\lambda \alpha) & \lambda \mathrm{Y}_{1}(\lambda \alpha)+h \mathrm{Y}_{0}(\lambda \alpha) \\
\mathrm{J}_{0}(\lambda) & \mathrm{Y}_{0}(\lambda)
\end{array}\right|=0,
$$


while for $\mathrm{BC} 3, \mathrm{R}(\alpha)=\mathrm{R}(1)=0$ giving

$$
\left|\begin{array}{cc}
\mathrm{J}_{0}(\lambda \alpha) & \mathrm{Y}_{0}(\lambda \alpha) \\
\mathrm{J}_{0}(\lambda) & \mathrm{Y}_{0}(\lambda)
\end{array}\right|=0 .
$$

For both $\mathrm{BC} 2$ and $\mathrm{BC} 3, \mathrm{~B}=-\mathrm{J}_{0}(\lambda) / \mathrm{Y}_{0}(\lambda)$.

These exact solutions have been checked using an Euler time-step finite different numerical scheme written in MATLAB. Second order central differences were used at all internal points with fictitious points outside the domain for derivative boundary conditions, hence allowing second order accuracy on the boundary. Care was taken to ensure the time step remained within stability limits. The solution was found accurate for a range of parameter values. In general, sufficient accuracy is achieved with a spatial discretisation of 21 points in both $r$ and $z$ directions. This numerical solution was used as a verification of our analytic results, as well as a tool for solving more complicated heat transfer scenarios which are the subject of further research; for example, when the radial diffusivity is a nonlinear function.

Of interest is the time taken for the cold point in the coil to reach a critical temperature. Continuing to work in scaled units we denote this time and temperature by $\tau_{\mathfrak{c}}$ and $\mathfrak{u}_{\mathfrak{c}}$, respectively. They are found numerically by solving Equation (16) implicitly for $\tau$ with $\mathfrak{u}=\mathfrak{u}_{\mathfrak{c}}$ at the cold point position: $z=1 / 2$ and $r=r_{c}$. The cold point position is estimated by finding the extremum of the leading eigenfunction $C_{0}\left(\lambda_{1} r\right)$, thus $r_{c}$ satisfies $C_{1}\left(\lambda_{1} r_{c}\right)=0$. Numerical tests confirm that for large times this $r_{c}$ is indeed at the cold point.

\section{Results}

Here, realistic values have been used with scaled parameters $\alpha=0.33, h=2$ and $\mathrm{D}=1$. Dimensionally we have $\mathrm{a}=0.25 \mathrm{~m}, \mathrm{~b}=0.75 \mathrm{~m}, \mathrm{~L}=1.1 \mathrm{~m}$, 


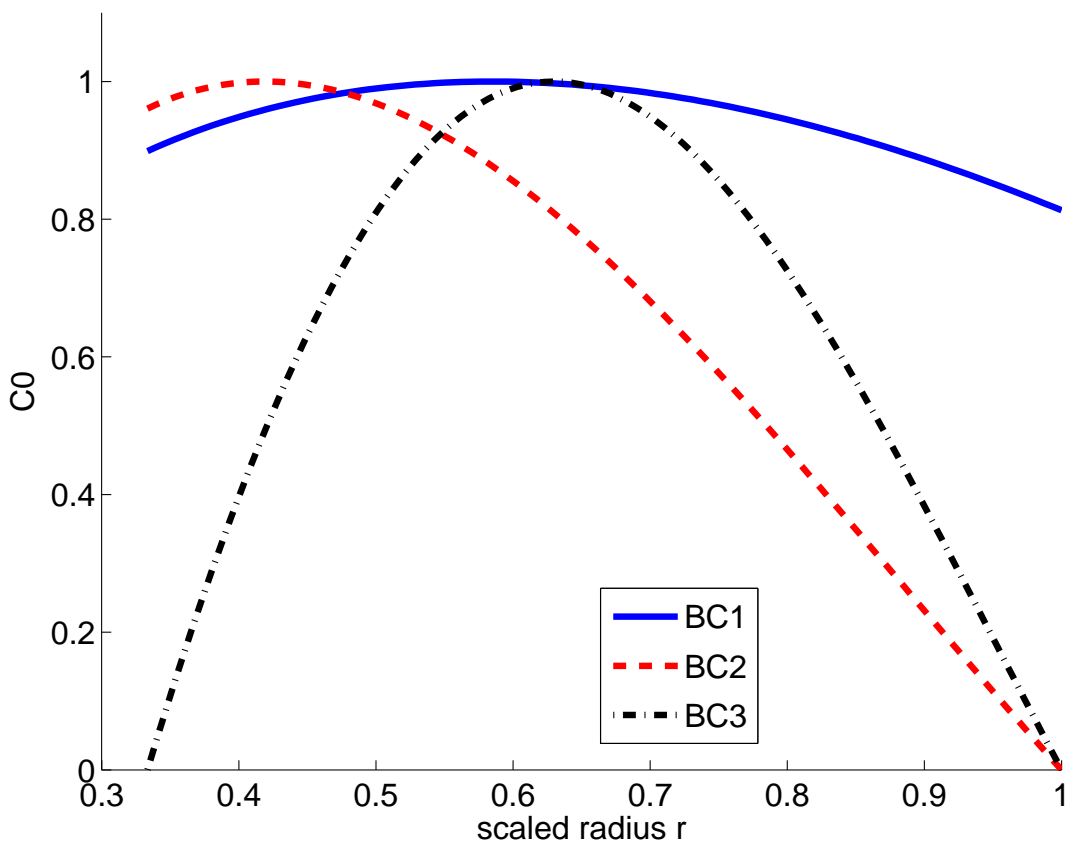

Figure 2: Leading eigenfunctions $C_{0}\left(\lambda_{1} r\right)$, Equation (14) scaled to a maximum of unity, for the three types of boundary conditions.

$c_{p}=1169 \mathrm{~J} / \mathrm{kg} / \mathrm{K}, \rho=7854 \mathrm{~kg} / \mathrm{m}^{3}, \mathrm{k}_{\mathrm{s}}=30 \mathrm{~W} / \mathrm{m} / \mathrm{K}$, and $\mathrm{H}=3.8 \mathrm{~W} / \mathrm{m}^{2} / \mathrm{K}$. Figure 2 shows the leading eigenfunction $C_{0}\left(\lambda_{1} r\right)$ for the three boundary conditions. This eigenfunction has the same approximate shape as the rescaled solution $\mathfrak{u}(r, z=0.5, \tau)$ for large enough time, and predicts the position of the cold point - the extremum of the eigenfunction. As expected, with BC2 the eigenfunction is far more asymmetric than with $\mathrm{BC} 1$ and $\mathrm{BC} 3$, producing a cold point close to the inner radius.

Figure 3 shows the scaled temperature at the cold point as a function of time. Also shown is the critical temperature $\boldsymbol{u}_{c}$ and the intersection points which give the time lag $\tau_{c}$. Note that with our scaling the temperature is 


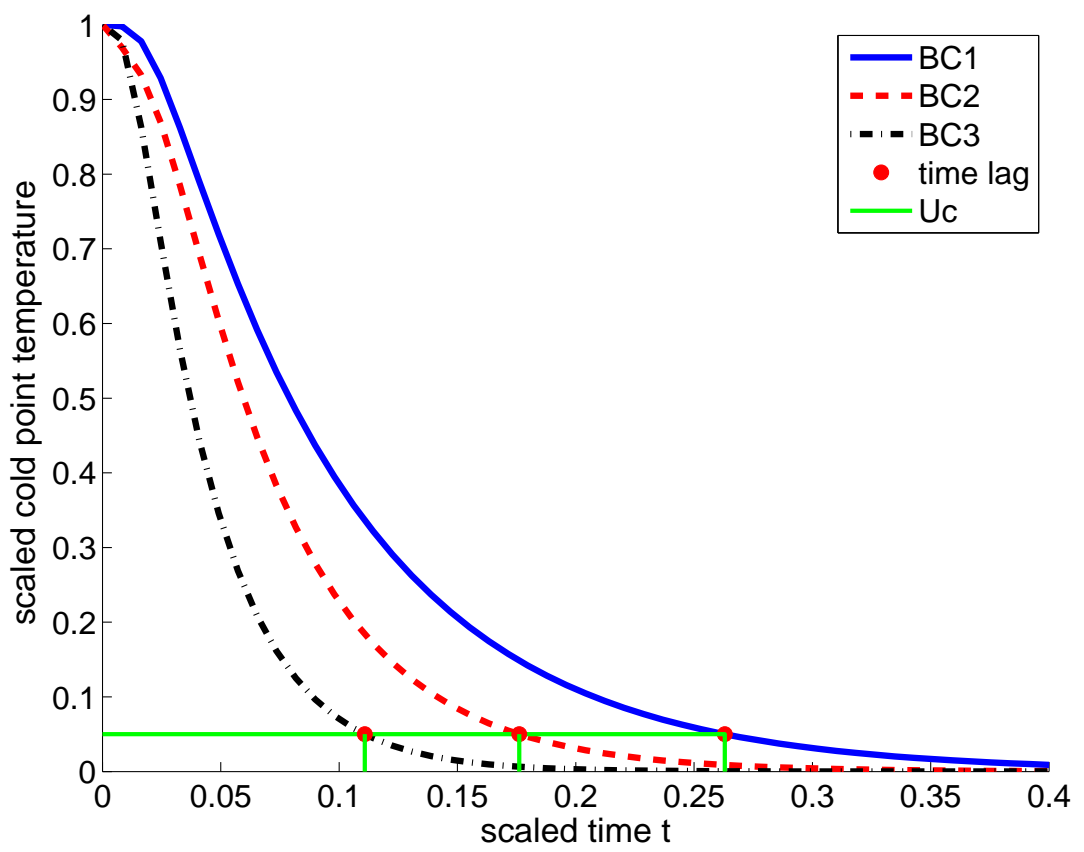

Figure 3: Cold point temperature as a function of time for the three types of boundary conditions.

cooling from $u=1$ to $u=0$. The value $\mathfrak{u}_{c}=0.05$ corresponds with an actual temperature $\mathrm{T}$ for which the coil has increased from $\mathrm{T}_{0}$ by $95 \%$ of the difference between $T_{0}$ and $T_{g}$. As expected $B C 1$, corresponding to Newton cooling on the curved surfaces, leads to the slowest decline in temperature. As shown by McGuinness et al. [7], the Newton cooling boundary condition is the limiting factor in the heat transfer, despite the large outer curved surface. With this boundary condition the heat transfer is vertically dominated hence making time lag relatively independent of $\alpha$ and D.

Figure 4 shows the time lag, $\tau_{\mathfrak{c}}$, as a function of the relative diffusivity for the same critical temperature value $\mathfrak{u}_{c}=0.05$. The radial diffu- 
sivity is a complex function of steel layer thickness, tension between layers and thermal expansion. Whilst this dependence is the subject of further research [5], the effective radial diffusivity is within the range $\mathrm{D}=0.2-1$ shown on this graph [8]. (The radial conductivity will be smaller than the steel conductivity, and hence the vertical conductivity, because of the air gaps between layers.) Figure 4 illustrates the smaller dependence of time lag on $\mathrm{D}$ with $\mathrm{BC} 1$, which arises because heat transfer is vertically dominated. Note that $\mathrm{D}=\mathrm{D}_{\mathrm{r}} \mathrm{L}^{2} / \mathrm{D}_{z} \mathrm{~b}^{2}$ which is dependent on cylindrical dimensions as well as effective radial diffusivity, $D_{r}$. Results presented here are for a fixed $h=\mathrm{Hb} / \mathrm{k}_{\mathrm{r}}=2$. Thus interpretation of the results with respect to dimensional variables must be done with care: if $k_{r}$ changes then both $h$ and $D$ would change.

If annealing operators are able to adjust their operating system so that the different type of boundary conditions are appropriate, this may come at an additional cost. Figure 4 provides a guide as to whether modifications could be cost effective. Consider the possibility of adding radiant heaters directly heating the coil through its outer curved surface (corresponding to $\mathrm{BC} 2$ ). If $\mathrm{D}=0.1$ the time lag for $\mathrm{BC} 1$ is $\tau=0.3239$ reducing to $\tau=0.3151$ for $\mathrm{BC} 2$, only a $2.7 \%$ reduction in time; thus the additional cost required to change the boundary conditions is unprofitable in this case. However, for $\mathrm{D}=1$ the reduction in time lag is from $\tau_{\mathrm{c}}=0.2630$ for $\mathrm{BC} 1$ to $\tau_{\mathrm{c}}=0.1761$ for $\mathrm{BC} 2$, a $33 \%$ reduction in time, possibly making this change cost effective.

\section{Conclusion}

The results presented here are a simple first model of the annealing process, but do allow estimates on the heating time to be calculated for three different applicable boundary conditions. The time lag, the time for the coldest point to reach a critical temperature, is of importance to an annealing operator. Determining the dependence of this time lag on coil dimensions, radial 


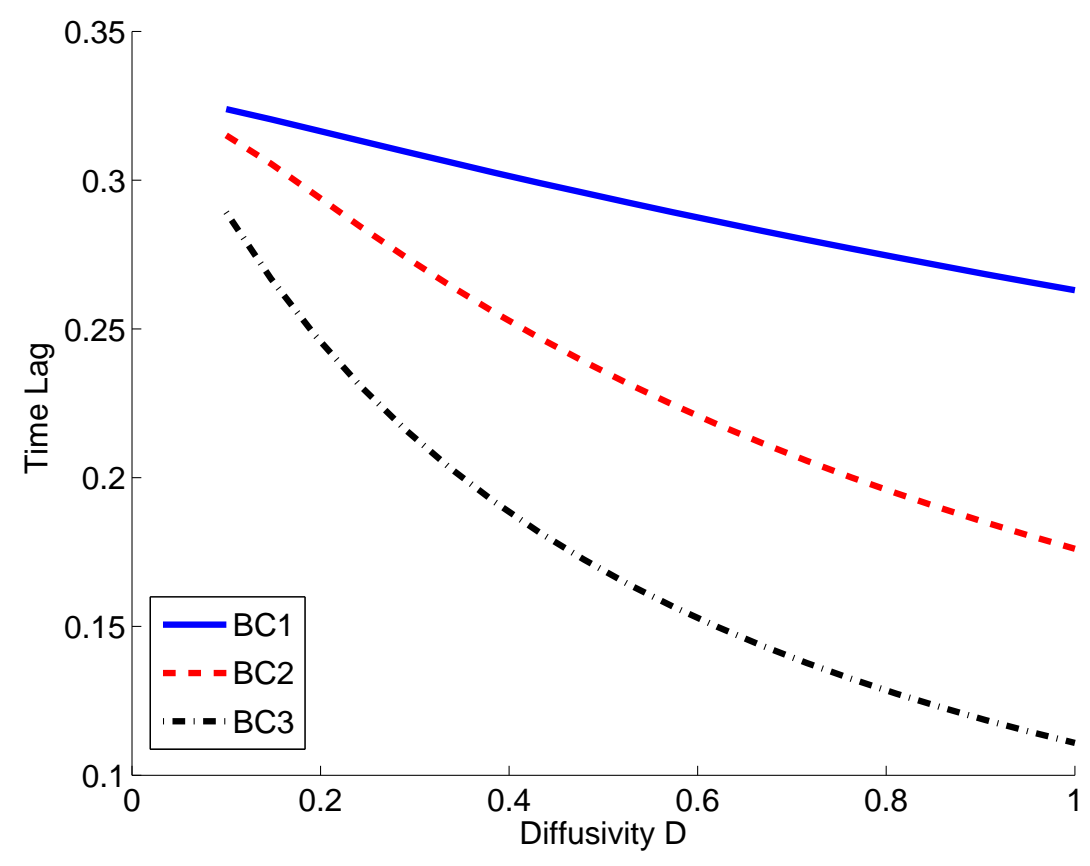

Figure 4: Time lag versus diffusivity $D$ for the three types of boundary conditions. 
diffusivity, gas temperature, and boundary conditions will help the steel industry to optimise production. Our further work will include models of the air gap, contact resistance, coil tension, coil expansion, anisotropic boundary conditions and incorporation of results shown by Hickson et al. [5].

\section{References}

[1] M. Abramowitz and I. Stegun. Handbook of Mathematical Functions, Dover, New York, 1970. http://mintaka.sdsu.edu/faculty/wfw/ABRAMOWITZ-STEGUN/ C673

[2] G. Chen and M. Gu. Simulation of steel coil heat transfer in a high performance hydrogen furnace. Heat Transf. Eng., 28, 25-30, 2007. doi:10.1080/01457630600985568 C671

[3] M. Cozijnsen and W. Y. D. Yuen. Stress distributions in wound coils. 2nd Biennial Austr. Eng. Math. Conf., 1996, 117-124. http://search.informit.com.au/documentSummary;dn= 716511683510327;res=IELENG C671

[4] G.F. Harvey. Mathematical simulation of tight coil annealing. Metallurgical forum 9, Math. Models, 22, 28-37, 1977. C671

[5] R. Hickson, S. I. Barry and G. N. Mercer. Heat transfer across multiple layers. ANZIAM J (E), submitted, 2008. C678, C680

[6] Y. Jaluria. Numerical study of the thermal processes in a furnace. Num. Heat Transf., 7, 211-224, 1984. doi:10.1080/01495728408961820 C671

[7] M. McGuinness, W. L. Sweatman, D. Baowan and S. I. Barry. Annealing Steel Coils. Proc. MISG 2008, in press, 2008. C669, C671, C672, C677 
[8] S. S. Sahay, A. M. Kumar and A. Chatterjee. Development of integrated model for batch annealing of cold rolled steel. Ironmaking and Steelmaking, 31, 144-151, 2004. doi:10.1179/030192304225010990 C678

[9] M. R. Sridhar and M. M. Yovanovicht. Review of elastic and plastic contact conductance models: Comparison with experiment.

J. Thermophysics Heat Transfer, 8, 633-640, 1994.

http: //www . aiaa.org/content. cfm?pageid=406\&gTable= japaperimportPre97\&gID=592 C670, C671

[10] U. O. Stikker. Numerical simulation of the coil annealing process, Math. Models in Metallurgical process development, Iron and Steel Institute, Special report. 123, 104-113, 1970. C670, C671

[11] W. Wu, F. Yu, X. Zhang and Y. Zuo. Mathematical model and its application of radial effective thermal conductivity for coil heat transfer in a HPH furnace. J. Thermal Science 11, 134-137, 2002. doi:10.1007/s11630-002-0033-1 C671

[12] X. Zhang, F. Yu, W. Wu and Y. Zhao. Application of radial effective thermal conductivity for heat transfer model of steel coils in HPH furnace. Int. J. Thermophysics, 24, 1395-1405, 2003.

doi:10.1023/A:1026115521232 C671

\section{Author addresses}

1. Steven I. Barry, University of New South Wales @ ADFA, Canberra, Australia. mailto:s.barry@adfa.edu.au

2. Winston L. Sweatman, Institute of Information and Mathematical Sciences, Massey University at Albany, Auckland, New Zealand. mailto:w. sweatman@massey . ac.nz 